\title{
Das Fragment einer Buchinschrift Philipp Melanchthons in der Andreas-Möller-Bibliothek Freiberg
}

\author{
von \\ HANS-PETER HASSE
}

Im Jahr 1931 publizierte der Freiberger Pfarrer Paul Schwen (1879-1949) in den „Beiträgen zur sächsischen Kirchengeschichte" sechs Bucheinträge Philipp Melanchthons, die in Bänden der deutschen Reihe der Wittenberger Luther-Ausgabe (Wittenberg 1551-1559) enthalten sind, die zur Kirchenbibliothek der Jakobikirche in Freiberg gehören. ${ }^{1}$ Von dem Eintrag Melanchthons im zweiten Band der Lutherausgabe (Wittenberg 1551) konnte Schwen nur den ersten Teil des Textes edieren, da er nur als Fragment überliefert ist. Schwen notierte dazu: „Das Vorsatzblatt mit dem Schluß des Beitrages fehlt. "2 Durch eine Recherche in der Andreas-Möller-Bibliothek des Geschwister-Scholl-Gymnasiums Freiberg ist es gelungen, das fehlende Blatt in einer Autografensammlung zu finden, die im 18. Jahrhundert der Freiberger Superintendent Christoph Gottlob Grundig (1707-1780) angelegt hatte. Sie gehört heute zu den Beständen der Andreas-Möller-Bibliothek. ${ }^{3}$ Da das Fragment der Handschrift Melan-

1 Paul Schwen, Handschriftliche Einträge Melanchthons in der Klosterbücherei St. Jakobi zu Freiberg, in: Beiträge zur sächsischen Kirchengeschichte 40 (1931), S. 17-27. Paul Schwen wirkte seit 1908 als Pfarrer an der Jakobikirche in Freiberg; vgl. zu den biografischen Daten Schwens ReInHOLd GRünbERG (Bearb.), Sächsisches Pfarrerbuch. Die Parochien und Pfarrer der Ev.-luth. Landeskirche Sachsens (1539-1939), Bd. 2: Die Pfarrer der Ev.-luth. Landeskirche Sachsens (1539-1939), Freiberg 1940, S. 862. Zur Kirchenbibliothek der Jakobikirche Freiberg vgl. KonRAD von Rabenau, Freiberg 5: Kirchenbibliothek der St. Jacobi-Gemeinde, in: Handbuch der historischen Buchbestände in Deutschland, Bd. 17: Sachsen, A-K, hrsg. von Friedhilde Krause, bearb. von Waltraut Guth/Dietmar Debes, Hildesheim/Zürich/New York 1997, S. 265-269. Seit Dezember 2012 befindet sich die Kirchenbibliothek als Depositum in der Bibliothek des Evangelisch-Lutherischen Landeskirchenamtes Sachsens in Dresden. Signatur der zwölf Bände der Wittenberger Lutherausgabe (deutsch, 1551-1559): Fol. 101-112; Bd. 1: Martin Luther: Der erste Teil der Bücher uber etliche Epistel der Aposteln. [Bd. 1] Wittenberg: Hans Lufft, 1551 [Signatur: Fol. 101]; Bd. 12: Der Zwelffte und letzte Teil der Bücher des Ehrnwürdigen herrn D. Mart. Lutheri [...]; Wittenberg 1559 [Signatur: Fol. 112]. Die deutsche Reihe der Wittenberger Lutherausgabe ist bibliografiert in: D. Martin Luthers Werke: kritische Gesamtausgabe (im Folgenden: WA), Bd. 60, Weimar 1980, S. 612-619; vgl. die Darstellung der Geschichte der Wittenberger Luther-Ausgabe von Eike Wolgast und Hans Volz: Eike Wolgast/Hans Volz, Geschichte der LutherAusgaben vom 16. bis zum 19. Jahrhundert, in: WA, Bd. 60, S. 464-495. Der Leiterin der Bibliothek des Landeskirchenamtes Dresden, Frau Susanne Liedke, danke ich sehr herzlich für ihre Unterstützung bei der Bearbeitung der Quellen und die Erstellung von Digitalisaten für den Abbildungsteil.

2 Schwen, Handschriftliche Einträge (wie Anm. 1), S. 19.

3 Andreas-Möller-Bibliothek des Geschwister-Scholl-Gymnasiums, Freiberg: Sammelband mit Autografen von Martin Luther, Philipp Melanchthon, Graf Nikolaus Ludwig 
chthons bislang nicht identifiziert und zugeordnet werden konnte, soll an dieser Stelle die fehlende Seite des Bucheintrages virtuell wieder in das Buch eingefügt werden, aus dem sie im 18. Jahrhundert entfernt wurde. Der aus den beiden Fragmenten rekonstruierte Text des Bucheintrages wird in diesem Beitrag ediert.

Leider lässt sich die Provenienz der Lutherausgabe mit den Bucheinträgen Melanchthons und damit auch des Fragmentes in der Andreas-Möller-Bibliothek nicht vollständig rekonstruieren. Der einzige Hinweis auf den Erstbesitzer sind dessen Initialen im Einband: „D P R“. Die Initialen finden sich in allen Bänden, die der Besitzer der Ausgabe in den Jahren zwischen 1552 (Band 1) und 1560 (Band 12) binden ließ. ${ }^{4}$ Zeitnah zum Erscheinen der einzelnen Bände legte er Melanchthon die Bände mit der Bitte um einen Eintrag vor. In sechs Bände schrieb Melanchthon im Zeitraum zwischen 1552 und 1556 Bucheinträge auf Spiegel und Vorsatzblätter der Bände. Dabei fällt auf, dass sich drei Bucheinträge inhaltlich ähneln, da Melanchthon dreimal die Bibelstelle Kolosser 3, 16 für einen Eintrag mit einer kurzen Auslegung des Bibelwortes wählte. Schwen hat bei seiner Edition der Bucheinträge (1931) versucht, anhand der Initialen den Erstbesitzer der Lutherausgabe zu finden, doch ist es ihm nicht gelungen. ${ }^{5}$ Er ver-

von Zinzendorf (1700-1760) und anderen Personen [ohne Signatur]. Der Sammelband wurde in den Jahren 2005 bis 2006 restauriert. Die Bindung wurde dabei gelöst und die Autografen in Einzelmappen eingelegt; vgl. den beiliegenden Restaurierungsbericht von Uwe Löscher. Dem Kustos der Andreas-Möller-Bibliothek, Herrn Dr. Volker Bannies, danke ich sehr herzlich für seine Unterstützung bei der Bearbeitung der Quellen und die Herstellung von Digitalisaten für den Abbildungsteil. Zur Geschichte der Gymnasialbibliothek vgl. die einschlägige Literatur in: VolkeR Bannies, Freiberger Bücherschätze: Andreas-Möller-Bibliothek. Mit Aufnahmen von Volkmar Herre, Stralsund/Beucha 2012, S. 136-142 [Literaturverzeichnis]. Ebd., S. 85 ist der Brief von Martin Luther an Kurfürst Johann den Beständigen vom 17. August 1529 reproduziert, der zu der Autografensammlung gehört. Zur Geschichte und zu den Beständen der Bibliothek vgl. ferner: Volker BANNiEs: Freiberg 2: Andreas-Möller-Bibliothek des Geschwister-Scholl-Gymnasiums, in: Handbuch der historischen Buchbestände in Deutschland (wie Anm. 1), Bd. 17, S. 248-256.

4 Das gilt nicht für den zehnten Band der Ausgabe (Signatur: Fol. 110), der einen anderen Einband aufweist und offenbar erst später dieser Ausgabe der Werke Luthers hinzugefügt wurde. Im Unterschied zu allen anderen Bänden ist nur dieser Band im Schnitt handschriftlich beschriftet (Schnitt unten: „Der Zehnt“; Schnitt längs: „X“). Ferner fehlt in diesem Band die handschriftliche Altsignatur auf dem Titelblatt, die sonst alle anderen Titelblätter der Lutherausgabe enthalten als Nummerierung von „Nr. 3165“ (Bd. 1) bis „Nr. 3176“ (Bd. 12); Bd. 10 würde die Signatur „Nr. 3174“ entsprechen, diese jedoch fehlt. Die Merkmale des zehnten Bandes sprechen dafür, dass er später erworben wurde, um wohl einen fehlenden Band zu ergänzen. Der Spiegel vorn enthält einen Kaufeintrag, dass der Band im Jahr 1711 in Freiberg von dem Kupferschmied Johann David Trülitzsch zu einem Preis von 15 Gulden erworben wurde; vgl. ScHwen, Handschriftliche Einträge (wie Anm. 1), S. 24 f.

5 Eine spätere Besitzerin der Lutherausgabe war „Rahel Helena Zeißin“, deren handschriftlicher Besitzvermerk auf den Titelblättern des vierten und siebenten Bandes zu finden ist (Signaturen: Fol. 104 und 107). Zwar gelang es nicht, die Identität von Rahel Helena Zeis zu klären, es gibt jedoch Hinweise, die vermuten lassen, dass Rahel Helena Zeis zur Familie des Schlettauer Pfarrers Christian Heinrich Zeis (1697-1761) gehörte, für die eine freundschaftliche Beziehung mit dem Freiberger Superintendenten Christoph Gottlob Grundig nachgewiesen ist. Das belegt eine Denkschrift für Christian Heinrich Zeis, zu der Grundig ein Gedicht beisteuerte. Christian Heinrich Valerius ZeIs, Erneuertes Andenken Herrn M. Christian Heinrich Zeisens, ehemaligen Pastors zu Schlettau, durch seinen älteren Sohn in Dresden, Dresden 1774. Vgl. zum Gedicht 
mutete den Besitzer in Wittenberg, konnte aber in den Daten der Matrikel und in den Promotionslisten der Wittenberger Universität keine Person finden, auf die die Initialen „D P R“ passen.6 Leider enthalten die Bände der Lutherausgabe keine Benutzerspuren, die zur Aufklärung der Provenienz etwas beitragen. Auf welchen Wegen sie nach Freiberg gelangte, ist nicht bekannt. Fest steht jedoch, dass die Lutherausgabe in der Mitte des 18. Jahrhunderts von August Gottfried Küchenmeister (1732-1810) und Johann Friedrich Rost (1732-1781) der Kirchenbibliothek der Freiberger Jakobikirche gestiftet wurde, an der sie als Pfarrer und Diakon tätig waren. ${ }^{7}$ Eine 1773 in Freiberg erschienene Publikation über „Bücherfeste“ liefert den Hinweis, dass die beiden Pfarrer der Jakobikirche und der Freiberger Superintendent Christoph Gottlob Grundig als Bibliophile das Interesse an Büchern und Bibliotheken pflegten. ${ }^{8}$ Als Küchenmeister 1810 starb, hinterließ er eine stattliche Bibliothek, die in einem Auktionskatalog beschrieben ist. ${ }^{9}$ Noch mehr gilt das für den Freiberger Superintendenten Grundig,

Grundigs: ebd., Bl. B 4r/v; digital zugänglich unter http://digital.slub-dresden.de/ ppn329661906/1 (Zugriff 25.08.2013). Der Großvater von Christian Heinrich Zeis war vermutlich Christian Zeis (1580-1636), der als Pfarrer in Liebschwitz wirkte und 1603 das Gymnasium Freiberg besucht hatte; für Daten zur Familie Zeis vgl. GrüNBERG, Sächsisches Pfarrerbuch (wie Anm. 1), Bd. 2, S. 1047. Die Beziehungen der Theologenfamilie Zeis nach Freiberg lassen vermuten, dass Rahel Helena Zeis, die zeitweise Besitzerin der Freiberger Lutherausgabe gewesen ist, zu dieser Familie gehörte.

6 Eine erneute Prüfung der Matrikel der Universität Wittenberg bestätigt den Befund. Für den Zeitraum vom 18. Oktober 1544 bis zum 8. August 1554 konnte kein Matrikeleintrag gefunden werden, auf den die Initialen „D P R“ passen; vgl. KARL EDUARD Förstemann (Hg.), Album Academiae Vitebergensis, Bd. 1: ab anno Christi MDII usque ad annum MDLX, Leipzig 1841, S. 217-297.

7 Nachweis der Stiftung von Küchenmeister und Rost anhand einer Eintragung in einem handschriftlichen Verzeichnis bei Schwen, Handschriftliche Einträge (wie Anm. 1), S. 24.

8 Anlässlich des Amtsjubiläums von August Theodor Küchenmeister (1702-1775), Pfarrer von Dorfhain und Klingenberg, gaben dessen beide Söhne Gottfried August, Pfarrer der Jakobikirche Freiberg, und Christian Theodor, Substitut des Pfarrers von Dorfhain und Klingenberg, gemeinsam mit dem Freiberger Superintendenten Grundig und dem Diakon der Jakobikirche Freiberg Johann Friedrich Rost eine Festschrift heraus, in der sie Beispiele für das Buch- und Bibliothekswesen in der Kirchengeschichte sowie Jubiläen berühmter Buchpublikationen (Luthers Bibelübersetzung, Augsburgische Konfession, Konkordienbuch u. a.) als „Bücherfeste“ vorstellten. Vgl. CHRIsTOPH Gottlob Grundig/GotTfried August Küchenmeister/Johann Friedrich Rost/ Christian Theodor Küchenmeister, Mit einigen Nachrichten von Bücherfesten. wollten Tit. Tot. Herrn August Theodor Küchenmeistern, Wolverordneten und bestverdienten Pastori Seniori zu Dorfhayn und Klingenberg, bey Dessen durch göttliche Gnade erlebten, und am V Sonntage nach Trinitatis, dieses 1773sten Jahres, begangenen Amtsjubelfeste, ihre kindliche und freundschaftliche Regungen und Wünsche darlegen [...]. Freiberg 1773; VD18: 10066241; digital zugänglich unter: vd18.de/id/645403 (Zugriff 25.08.2013).

9 Die Bibliothek Küchenmeisters umfasste 2.246 Bände; vgl. Verzeichniß der Bibliothek des Hrn. Aug. Gottfr. Küchenmeister, weil. Amtspredigers zu St. Nicolai in Freyberg, welche den 3. Dezember 1810 und folgende Tage in dessen ehemaliger Wohnung par terre [...] verauctionirt werden sollen, Freiberg 1810. Ein Exemplar liegt in der Sächsische Landesbibliothek - Staats- und Universitätsbibliothek Dresden (SLUB), Signatur: 2.A.4505. Auf der Rückseite des Titelblattes findet sich eine Notiz, die die bibliophilen Interessen Küchenmeisters dokumentiert: In dem literarischen Nachlaße des verstorbenen Hern Amtsprediger Küchenmeister in Freyberg fanden sich, außer seiner Bibliothek: 1) eine Sammlung von Landcharten, welche in 4 Bänden und einem Faszikel 
der selbst publizierte und über eine hervorragend ausgestattete Gelehrtenbibliothek verfügte. Dass er daneben auch Autografen von Reformatoren und anderen berühmten Theologen sammelte, zeigt der Sammelband von Autografen, zu dem das Fragment des Bucheintrages von Melanchthon gehört. Reste seiner Bibliothek sind heute noch in Freiberg erhalten, erkennbar an dem signifikanten Exlibris, mit dem auch der Autografenband versehen ist: ein aufgeschlagenes Buch mit kleinen Blüten und einem Zitat Vergils: Tantus amor florum (Groß ist die Liebe zu den Blumen). ${ }^{10}$

Christoph Gottlob Grundig wurde 1707 als Sohn des Pfarrers Georg Gottlob Grundig (1671-1731) in Dorfhain im Osterzgebirge geboren. ${ }^{11}$ Nach dem Besuch der Kreuzschule in Dresden (1717-1719) und des Gymnasiums in Freiberg (1722-1727) studierte er an der Universität Leipzig Philosophie, Geschichte, Sprachen und Theologie und wurde 1731 zum Magister artium promoviert. Er wirkte als Hauslehrer in Eisleben und seit 1733 in Dresden, bis er 1737 eine Pfarrstelle in Hermannsdorf (bei Annaberg) übernahm. 1749 wurde er Pfarrer in Schneeberg, 1758 Superintendent in Glauchau und 1759 Superintendent in Freiberg, wo er auch als Schulinspektor wirkte und die Gründung der Bergakademie Freiberg (1765) unterstützte. Grundig trat durch zahlreiche Veröffentlichungen hervor, darunter mehrere theologische Schriften und die Monatszeitschrift „Versuche nützlicher Sammlungen zur Natur- und Kunstgeschichte sonderlich von Obersachsen“ (1746-1761). Sein Andachts- und Liederbuch

einzelner Blätter weit über 400 Stücke in sich begreift; 2) eine Sammlung von mehr als 300 gedruckten Autographis Lutheri, (wovon sich in einigen selbst seine Handschrift befindet,) und einiger seiner Zeitgenoßen, aus 26 Faszikeln bestehend, in Capseln; 3) eine nicht geringe Sammlung von größtentheils älteren Disputationen und Programmen, ebenfalls in Capseln. Von diesen beiden letzern Sammlungen ist auch ein geschriebener Catalog vorhanden. Über den Verbleib der Autografen Martin Luthers und anderer Reformatoren aus der Bibliothek Küchenmeisters ist nichts bekannt. Die Notiz in dem Auktionskatalog beweist, dass in dem Kreis der Freiberger Bibliophilen um Grundig und Küchenmeister auch Handschriften der Reformatoren gesammelt wurden.

10 Siehe Abb. 1: Exlibris der Bibliothek von Christoph Gottlob Grundig; Abb. nach dem Exlibris in dem Autografenband (wie Anm. 3). Grundig verwendete in Verbindung mit dem Exlibris ein gedrucktes Zitat von Lactantius mit seinen Initialen und der Datierung 1731: Lactantius de opific[io] Dei c. XXI. in fine. Quod si vita est optanda Sapienti, profecto nullam aliam ob causam vivere optaverim, quam ut aliquid efficiam quod vita dignum sit. [...] Quo perfecto satis me vixisse arbitrabor et officium hominis implesse, si labor meus aliquos homines ab erroribus liberatos, ad iter caeleste direxerit. („Wenn der Weise sich das Leben wünschen soll, so möchte ich wahrlich aus keinem andern Grunde zu leben wünschen, als um ein Werk zu vollbringen, das des Lebens wert wäre, [...]. Gelingt mir dies, so glaube ich genug gelebt und meine Pflicht als Mensch erfüllt zu haben, wenn ich einige Menschen vom Irrtum befreit und ihnen den Weg zum Himmel gewiesen habe.“; Übersetzung von Aloys Hartl, zitiert nach Des Lucius Caelius Firmianus Lactantius Schriften. Auszug aus den Göttlichen Unterweisungen, aus dem Lateinischen übersetzt von Aloys Hartl (Bibliothek der Kirchenväter 1/36), Kempten/ München 1919, S. 284. Die Stellenangabe Grundigs zum Werk des Laktanz ist zu korrigieren: Lactantius, De opificio dei, Cap. 20, 8; vgl. Samuel Brandt/Georg Laubmann (Hg.), L. Caeli Firmiani Lactanti opera omnia (Corpus scriptorum ecclesiasticorum latinorum 37), Prag/Wien/Leipzig 1894, S. 64, 11-13.16-18 (Auslassung im Zitat).

11 Zur Biografie Grundigs vgl.: Denkmal Herrn M. Christoph Gottlob Grundig, Pastoris primarii, Superintendenten und Schulinspectoris zu Freyberg, Freiberg 1781; GABRIELE Meissner, Fußnote: *) s.M.C.G. Grundig, in: Sächsische Heimatblätter 49 (2003), S. 151-158; Dies., Christoph Gottlob Grundig 1707-1780, Dorfhain 2002; Dies., Superintendent Mag. Christoph Gottlob Grundig, in: Der Anschnitt 62 (2010), S. 261-266. 
„Geistlicher Bergbau“ (1750) wurde wiederholt aufgelegt. Berühmt wurde seine Bibliografie der nach dem Erdbeben von Lissabon (1755) erschienenen Publikationen zu diesem Ereignis. Mit seinem Tod am 9. August 1780 hinterließ er eine stattliche Bibliothek und eine wertvolle Mineraliensammlung. Ein Teil seiner Bibliothek wurde der Kirchenbibliothek der Jakobikirche in Freiberg gestiftet. ${ }^{12}$

Die Frage, wann das Melanchthonautograf in den Besitz Grundigs und damit in dessen Autografensammlung gelangte, lässt sich heute nicht mehr beantworten. Vermutlich haben dabei die Kontakte Grundigs zu seinen Kollegen Küchenmeister und Rost an der Jakobikirche eine Rolle gespielt.

Nach Inhalt und Form entspricht der Bucheintrag Melanchthons im zweiten Band der Wittenberger Lutherausgabe der Gattung der Buch- und Stammbucheinträge, die an der Wittenberger Universität entstand, weil Studenten die Reformatoren um handschriftliche Eintragungen in ihre Bücher baten. Sowohl von Luther als auch von Melanchthon sind zahlreiche Bucheinträge bekannt und ediert. ${ }^{13}$ Entsprechend den Merkmalen der Gattung schrieb Melanchthon zuerst ein Bibelwort in das Buch (Kolosser 3, 16), an das er eine kurze Auslegung anschloss mit Datum und Unterschrift. Die Bibelstelle Kol 3, 16 gehört zu den bevorzugten Zitaten, die Melanchthon für Bucheinträge nutzte. Das zeigt auch die Lutherausgabe der Freiberger Jakobikirche, in der die Bibelstelle in zwei weiteren Bucheinträgen zu finden ist. Ein Bucheintrag Melanchthons mit dem Spruch Kol 3, 16 ist in der Sammlung von Bibeleinträgen Wittenberger Reformatoren enthalten, die Georg Rörer (1492-1557) in Wittenberg unter dem Titel „Vieler schönen Sprüche aus Göttlicher Schrifft auslegung / daraus Lere und Trost zu nemen / Welche der ehrwirdige Herr Doctor Martinus Luther seeliger / vielen in jre Biblien geschrieben. Dergleichen Sprüche von andern Herrn ausgelegt / sind auch mit eingemenget" (1547) herausgab. ${ }^{14}$ Melanchthons sentenzartige Auslegung des Bibelwortes Kol 3, 16 in seinen Bucheinträgen zielt darauf, den Leser und Besitzer des betreffenden Buches aufzufordern, an der Lehre Jesu Christi festzuhalten und das in der Bibel überlieferte Wort Gottes zu studieren. ${ }^{15}$

12 Vgl. Rabenau, Freiberg 5 (wie Anm. 1), S. 266. Eine Zusammenstellung, welche Bände der Kirchenbibliothek der Jakobikirche aus der Bibliothek Grundigs stammen, gibt es bislang nicht. Gabriele Meißner weist darauf hin, dass Bände aus der Bibliothek Grundigs im Nachlass des Freiberger Mineralogen und Montanwissenschaftlers Abraham Gottlob Werner (1749-1817) enthalten sind, ohne dazu nähere Angaben zu machen; Meissner, Christoph Gottlob Grundig (wie Anm. 11), S. 14. Zudem wird von Meißner festgestellt, dass zur Bibliothek Grundigs 4.000 Bände gehörten. Die Bände aus der Bibliothek Grundigs im Nachlass Werners wären im wissenschaftlichen Altbestand der Universitätsbibliothek Freiberg zu suchen, wo der handschriftliche Nachlass Werners, seine Privatbibliothek sowie seine Sammlung von Rissen, Karten und Münzen erhalten sind.

13 Vgl. dazu die Nachweise und Literatur bei: Hans-Peter Hasse, Melanchthon und die „Alba amicorum“. Melanchthons Theologie im Spiegel seiner Bucheintragungen, in: Günter Frank (Hg.), Der Theologe Melanchthon (Melanchthon-Schriften der Stadt Bretten 5), Stuttgart 2000, S. 291-338; Ders., Wittenberger Theologie im „Stammbuch“. Eintragungen Wittenberger Professoren im Album des Wolfgang Ruprecht aus Eger, in: Michael Beyer/Günther Wartenberg (Hg.), Humanismus und Wittenberger Reformation. Festgabe anlässlich des 500. Geburtstages des Praeceptor Germaniae Philipp Melanchthon am 16. Februar 1997, Leipzig 1996, S. 88-120.

14 Bibliografische Beschreibung der Ausgabe in: WA, Bd. 48, Weimar 1927, S. XLIII mit RN S. 25; vgl. das Titelblatt der Erstausgabe bei Hasse, Melanchthon und die „Alba amicorum“ (wie Anm. 13), Abb. 4; dazu die Ausführungen ebd. S. 293 und 301.

15 Dass das Bibelwort Kol 3, 16 für Melanchthon eine zentrale Kernstelle der Heiligen Schrift gewesen ist, zeigt auch die Tatsache, dass er über dieses Bibelwort eine Rede ver- 


\section{Edition des Bucheintrages}

Der Edition des Textes werden die beiden Fragmente der Handschrift Philipp Melanchthons zugrunde gelegt:

Fragment 1: Bucheintrag Melanchthons auf dem vorderen Spiegel des zweiten Bandes der Lutherausgabe:

Fundort: Freiberg, Kirchenbibliothek der Jakobikirche: Signatur: Fol. 102; zurzeit Depositum in Dresden, Bibliothek des Evang.-Luth. Landeskirchenamtes Sachsens. Vgl Abb. 3.

Das Fragment ist ediert bei Schwen, Handschriftliche Einträge (wie Anm. 1), S. 19.

Fragment 2: Vorsatzblatt der Lutherausgabe mit der Handschrift Melanchthons auf der Vorder- und Rückseite. Das Einzelblatt gehört zu einer Autografensammlung, die im 18. Jahrhundert Christoph Gottlob Grundig gehörte (Exlibris; Abb. 1).

Fundort: Freiberg, Andreas-Möller-Bibliothek des Geschwister-Scholl-Gymnasiums: ohne Signatur.

Vgl. Abb. 4 und 5.

\section{[Fragment 1]}

Paulus zu den I Colossern cap. 3. I

Die rede des Herrn Christi soll | in euch wohnen reichlich | in aller weißheit, und soll | euch unter einander lehren I vnd erinn[ern]16 |

In disem schonen spruch sind viel I nottiger erinnerung, wie wol I mit kurtzen worten, doch I mit grossem ernst [ge] fasst ${ }^{17}$, |

Zum ersten, das wir Gott also I und nicht anders erkennen mogen, I nemlich durch die reden Christi, I das ist durch sein Euangelium.

[Fragment 2 = Vorsatzblatt recto]

Zum andern, das derhalben Gottes I ernstlich vnd vnwandelbar gepott I ist, das wir die selbige lehr I selb offt vnd viel betrachten, denn I ehr spricht: sie soll reichlich in I vns wohnen, das ist sie soll vns I nicht ein frembder gast sein, I sondern soll vns wol bekant sein I wie vnsere beiwohner. I Vnd ist gewißlich war, wo das hertz I gottes wort betracht vnd mit I glawben annimmet, da wohnet I gewisslich Gott selb im hertzen, I gebet sein liecht, heiligen geist, I liebe, trost, frewd zu rechter I anruffung, gehorsam vnd I ewigem leben.

Zum dritten spricht Paulus, die rede des I [Vorsatzblatt verso] herrn Christi soll in vns | wohnen, in aller weisheit, | das ist wir sollens recht | verstehen, wie sie | gott selb erkleret, sollen I nicht eigen deutungen | tichten, darumb soll | [... $]^{18}$ vleissig betrachten

fasste, die von Georg Major bei einer Promotion an der Universität Wittenberg am 2. Oktober 1550 vorgetragen wurde: [Philipp Melanchthon u. a.], Oratio de dicto ad Colossenses. Sermo Christi habitet in vobis abunde in omni sapientia, docentes \& admonentes vos. Recitata a Georgio Maiore in promotione nobilis \& clarißimi viri Domini Georgii Veneti Theologiae Doctoris, Wittenberg 1550; bibliografiert und beschrieben in: Horst Koenn, Philipp Melanchthons Reden. Verzeichnis der im 16. Jahrhundert erschienenen Drucke (Archiv für die Geschichte des Buchwesens 25), Frankfurt a. M. 1984, Sp. 1369 (Nr. 167). Der Text der Rede Melanchthons ist ediert in: Karl Gottlieb Bretschneider (Hg.), Philippi Melanthonis opera quae supersunt omnia (Corpus Reformatorum 11), Halle 1843, Sp. 895-901 (Nr. 113).

16 Kolosser 3, 16. Textverlust durch Abrieb im Wort „erinn[ern]“.

17 Textverlust durch Abrieb im Wort „[ge]fasst“.

18 Textverlust durch Abrieb. 
| vnd [alle stellen] ${ }^{19}$ ordenlich | gegen einander halden und I gott vmb verstand bitten | wie Dauid spricht. I Psal. 109 | Herr thue I barmhertzigkeit an deinem I knecht, vnd lehre mich | deine lehr. ${ }^{20}$

1552

Scriptu[m] manu Philippi
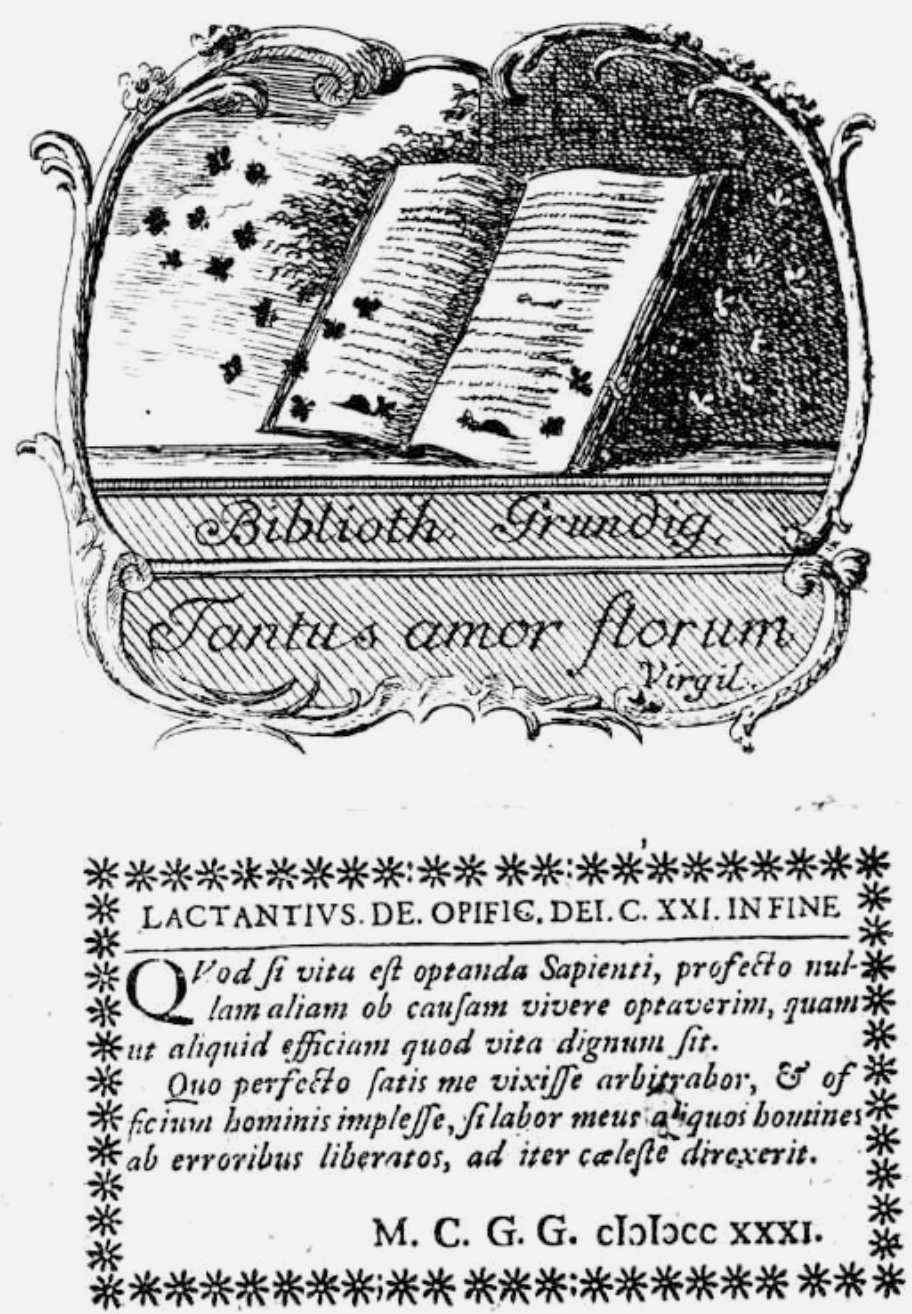

Abb. 1: Exlibris von Christoph Gottlieb Grundig.

19 Textverlust durch Abrieb; die in eckigen Klammern gebotene Lesart ist ein Vorschlag zur Rekonstruktion des Textes.

20 Das Zitat lässt sich nicht als Zitat von Psalm 109 verifizieren. Es könnte als ein Mischzitat gelesen werden aus 1. Samuel 20, 8 a und Psalm 119, 26b; vgl. auch Psalm 119, $65 \mathrm{f} ., 77,124 \mathrm{f}$. 


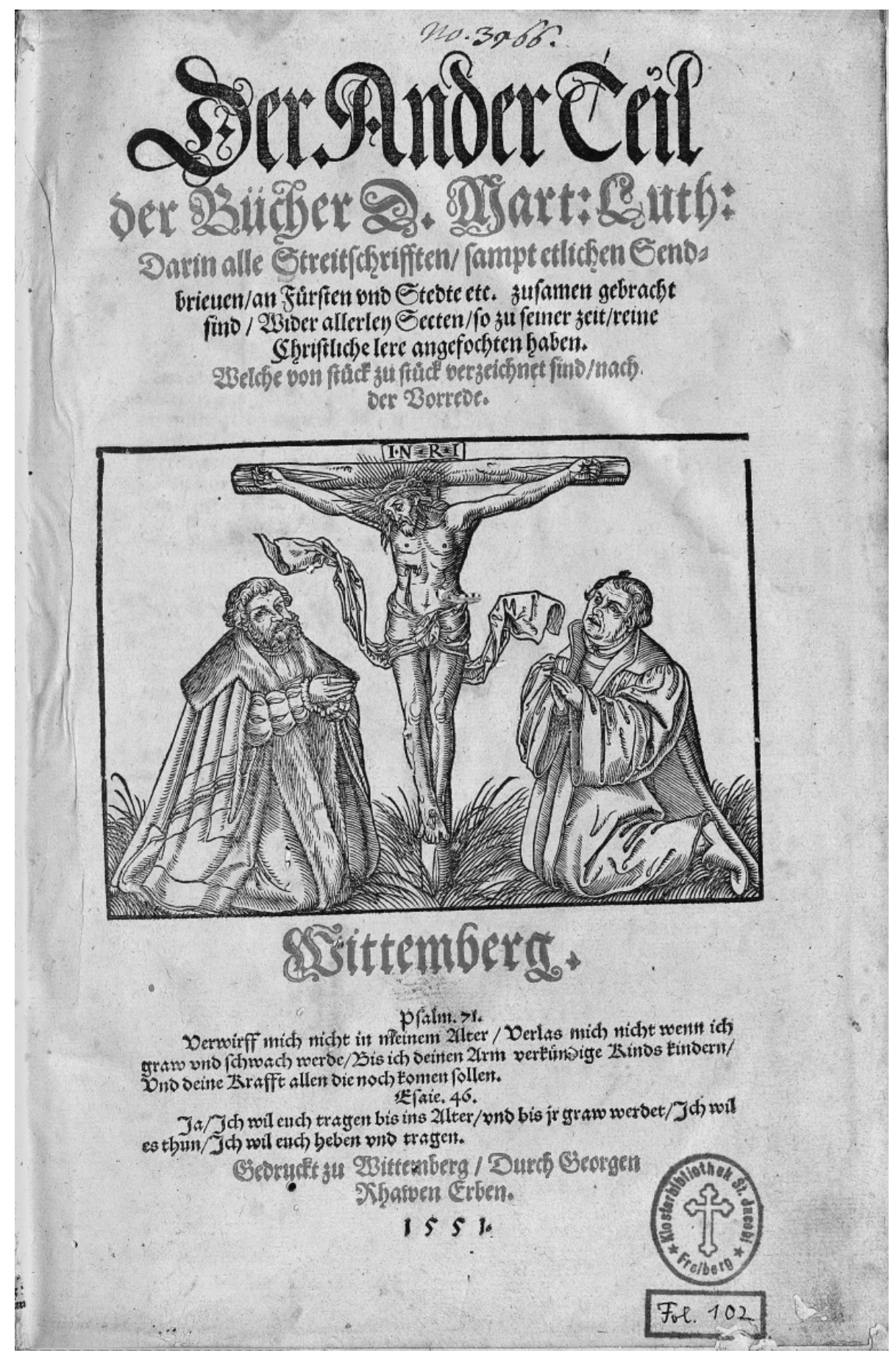

Abb. 2: Titelblatt des 2. Bandes der Wittenberger Lutherausgabe, 1551. 


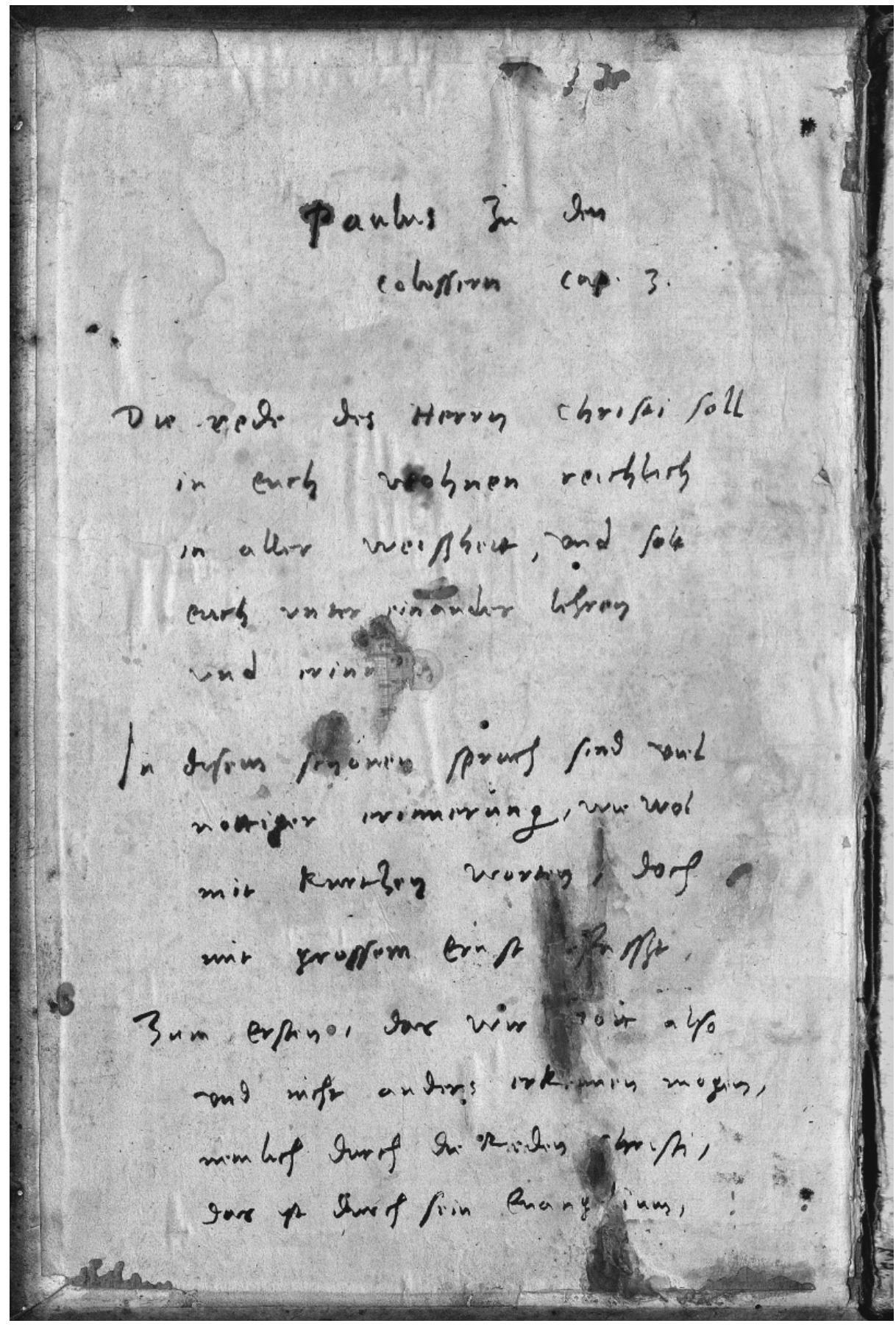

Abb. 3: Bucheintrag Melanchthons auf dem vorderen Spiegel des 2. Bandes der Wittenberger Lutherausgabe, [1552]. 


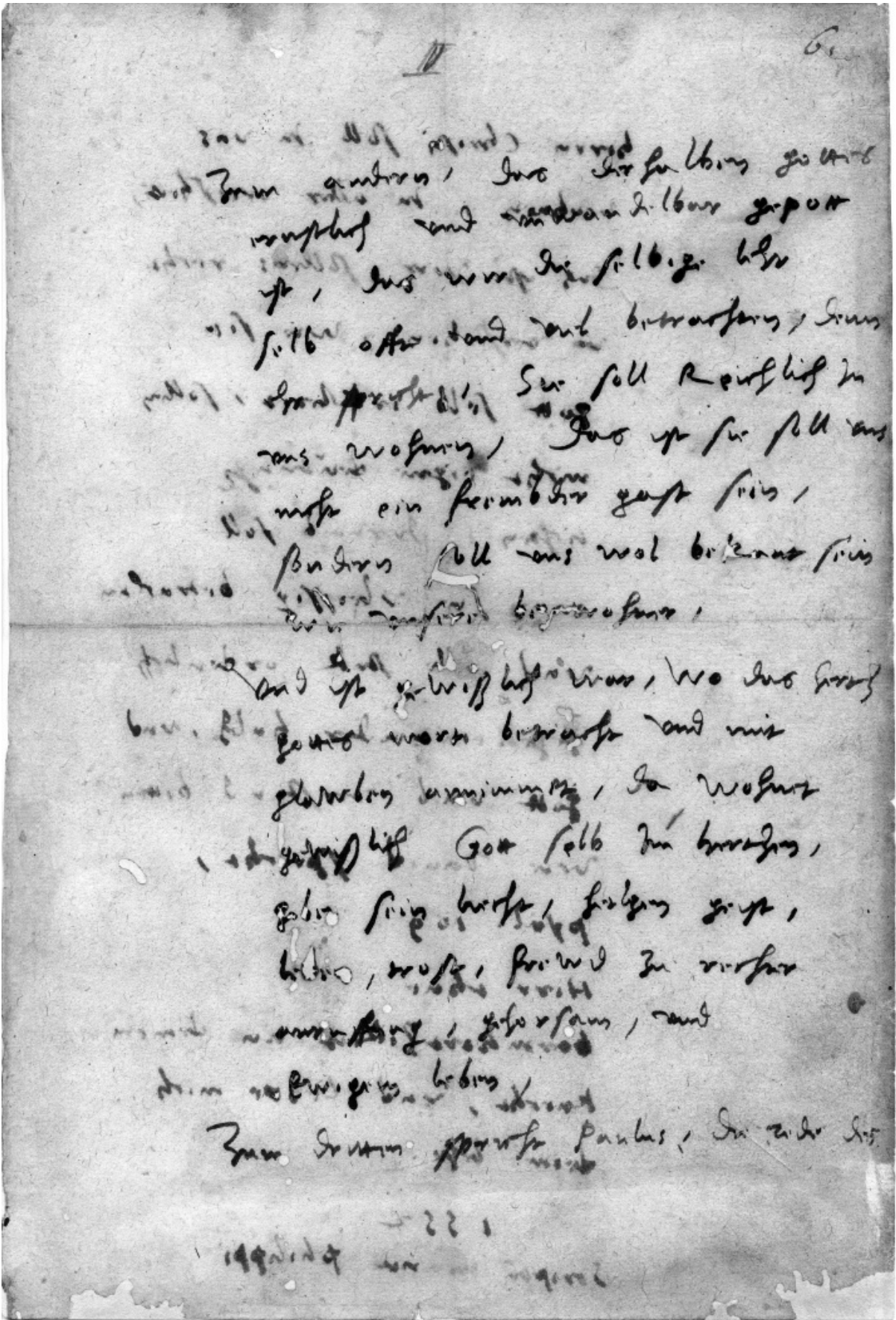

Abb. 4: Bucheintrag Melanchthons auf dem Vorsatzblatt (recto) des 2. Bandes der Wittenberger Lutherausgabe, [1552]. 


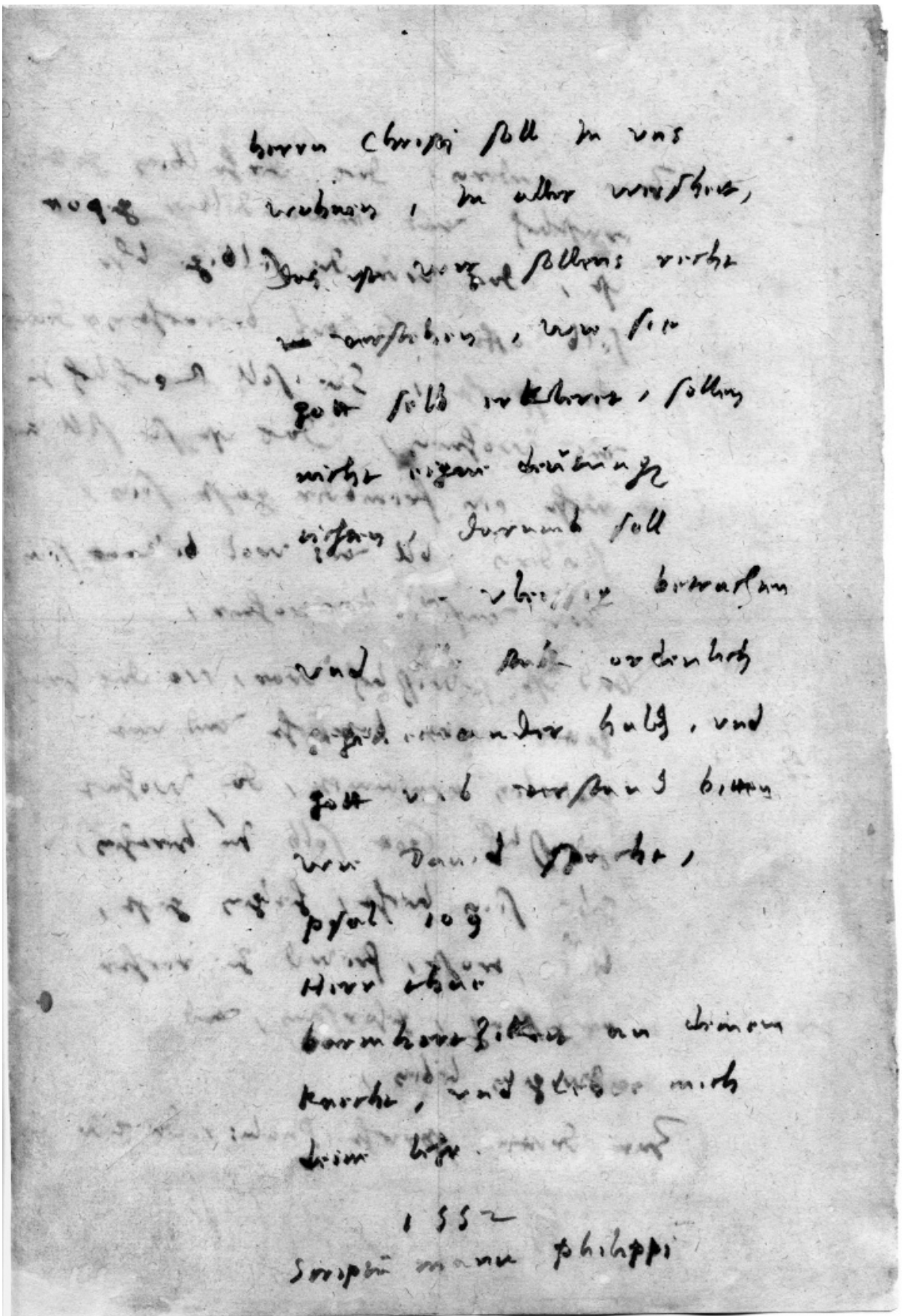

Abb. 5: Bucheintrag Melanchthons auf dem Vorsatzblatt (verso) des 2. Bandes der Wittenberger Lutherausgabe, [1552]. 